\title{
CREATIVITY AND EFFECTIVE INSEPARABILITY
}

\author{
BY \\ RAYMOND M. SMULLYAN(1)
}

Introduction. Our terminology is from [1]. We consider the collection of all r.e. (recursively enumerable) sets arranged in an effective sequence $\omega_{1}, \omega_{2}, \cdots, \omega_{i}, \cdots$ as in the Post-Kleene enumeration, i.e., $\omega_{i}$ is the set of all numbers satisfying the condition $(\exists y) T_{1}(i, x, y)$, where $T_{1}^{\prime}(z, x, y)$ is the Kleene predicate (cf. [2]). A number set $\alpha$ is called productive iff there exists a recursive function $f(x)$ (called a productive function for $\alpha$ ) such that for every number $i$, if $\omega_{i} \subseteq \alpha$ then $f(i) \in \alpha-\omega_{i}$. A number set $\alpha$ is called creative iff $\alpha$ is r.e. and the complement $\tilde{\alpha}$ of $\alpha$ is productive. A disjoint pair $(\alpha, \beta)$ of number sets is called recursively inseparable (abbreviated "R.I.") iff there exists no recursive superset of $\alpha$ which is disjoint from $\beta$. The pair $(\alpha, \beta)$ is effectively inseparable (abbreviated 'E.I.') iff there is a recursive function $\delta(x, y)$-called an E.I. function for the pair $\alpha, \beta$-such that for every disjoint pair $\left(\omega_{i}, \omega_{j}\right)$ of respective r.e. supersets of $(\alpha, \beta)$, the number $\delta(i, j)$ is outside both $\omega_{i}$ and $\omega_{j}$. It has been independently shown by Trahtenbrat and by Tennenbaum that there exists an R.I. pair $(\alpha, \beta)$ of r.e. sets which is not E.I.

In this paper we obtain some new characterizations of creativity and effective inseparability (as well as some closely allied notions) which seem surprisingly weak. These results are in some respects stronger than those obtained in [1]. The characterizations arose from the consideration of the following metamathematical problem.

Consider a first order theory $(T)$ with standard formalization (cf. Tarski [3]) and an effective Gödel numbering $g$ of the expressions of $(T)$. We let $T_{0}, R_{0}$, respectively, be the set of Gödel numbers of the provable and refutable sentences of $(T)$ [by a refutable sentence we mean one whose negation is provable]. We refer to these 2 sets as the nuclei of (T). For the time being we shall consider only those theories $(T)$ which are axiomatizable-i.e., such that $T_{0}$ (and hence also $R_{0}$ ) is an r.e. set. A formula $F(x)$ (with just one free variable $x$ ) is said to represent in (T) a number set $A$ iff $F(x)$ is provable for every $n \in A$ but for no $n \notin A-$ i.e., for every $n, n \in A$ iff $F\left(\Delta_{n}\right)$ is provable. Myhill [4] showed that if every r.e. set is representable in $(T)$ then $(T)$ is undecidable-i.e., $T_{0}$ is not recursive. We have shown $[5 ; 1]$, that a sufficient condition for $(T)$ to be undecidable is that every recursive set be representable in $(T)$. However, under Myhill's stronger hypothesis that every recursively enumerable set is representable in $(T)$, the set $T_{0}$ is not

Received by the editors October 15, 1962.

(1) This research was supported in part by a grant from the Air Force Office of Scientific Research. 
only nonrecursive, but in fact creative $\left({ }^{2}\right)$. On the other hand, if just the recursive sets are representable in $(T)$, then $(T)$ is not necessarily creative-i.e., $T_{0}$ is not necessarily a creative set. This was shown by Schoenfield [6]. In [1] we introduced the notion of all recursive sets being uniformly representable in $(T)$. Roughly speaking, this means that given any index of a recursive set, we can effectively find a formula of $(T)$ to represent it. Precisely speaking, it means that there is a (general) recursive function $f(x)$ such that for every number $i$, if $\omega_{i}$ is recursive, then $f(i)$ is the Gödel number of a formula of $(T)$ which represents $\omega_{i}$. We showed in [1] that if all recursive sets are uniformly representable in $(T)$, then $(T)$ (if axiomatizable) is creative.

The above remarks were concerned with questions of representing number sets within theories. A formula $F(x)$ is said to define a number set $\alpha$ within $(T)$ if $F$ is provable for every $n \in \alpha$ and refutable for every $n \notin \alpha$. And $\alpha$ is definable in $(T)$ if there is a formula $F(x)$ which defines it in $(T)$. [For a consistent theory, definability implies representability, but not conversely (unless the theory is also complete).] Consider now a consistent axiomatizable theory $(T)$ in which all recursive sets are definable. Putnam [7] has shown that such a theory must be essentially undecidable-i.e., that every consistent extension is undecidable. We showed [5] the stronger fact that for such a theory $(T)$, the pair $\left(T_{0}, R_{0}\right)$ must be recursively inseparable. For such a theory $(T)$, is the pair $\left(T_{0}, R_{0}\right)$ necessarily effectively inseparable? The answer is "no" for the following reasons. The theory $(T)$ constructed by Shoenfield [6] is consistent, axiomatizable, and is such that every recursive set is not only representable in $(T)$, but definable in $(T)$. And yet the set $T_{0}$ is not creative. A fortiori, the pair $\left(T_{0}, R_{0}\right)$ is not effectively inseparable $\left({ }^{3}\right)$. So this particular pair is recursivelyinseparable by [6] but not effectively inseparable. [This argument, incidentally, affords an alternative method of constructing a recursively inseparable pair of r.e. sets which is not effectively inseparable.]

Let us now say that all recursive sets are uniformly definable in $(T)$ if there is a recursive function $g(x, y)$ such that for all numbers $i, j$, if $\omega_{j}$ is the complement of $\omega_{i}$, then $g(i, j)$ is the Gödel number of a formula which defines $\omega_{i}$ in $(T)$. [Intuitively, this means that given any index of a recursive set $\alpha$, as well as an index of its complement, we can effectively find a formula of $(T)$ which defines $\alpha].\left({ }^{4}\right)$ Now, the metamathematical problem we wish to consider is this: if $(T)$ is a

(2) Without the assumption of axiomatizability, it is still true that if every r.e. set is representable in $(T)$, then $T_{0}$ is productive.

(3) It is a relatively trivial matter to verify that if $(\alpha, \beta)$ is an E. I. pair of r.e. sets, than $\alpha, \beta$ must both be creative. A proof can be found, e.g. in [8] - paragraph 4.

(4) The reader might wonder why we require that we be given an index of the complement of $\alpha$ as well as an index of $\alpha$. The fact is that there is no consistent theory $(T)$ with the stronger property that there exists a recursive function $f(x)$ such that for every number $i$ for which $\omega_{i}$ is recursive, $f(i)$ is the Gödel number of a formula which defines $\omega_{i}$ in $(T)$. If there were such a theory, then there would be a recursive function $g(x)$ such that for all $i$ for which $\omega_{i}$ is recursive, $g(i)$ is an index of $\tilde{\omega}_{i}$. And this is known to be impossible. The author is indebted to John Myhill for this observation. 
consistent theory in which all recursive sets are uniformly definable, is the pair $\left(T_{0}, R_{0}\right)$ necessarily effectively inseparable? We answer this question affirmatively. We in fact prove the following more general statement which is purely recursive function-theoretic in nature. "A sufficient condition for a disjoint pair $(\alpha, \beta)$ to be E.I. is that the collection of $\Sigma$ of all complementary pairs of recursive sets is uniformly reducible to $(\alpha, \beta)$, as defined in [1]-i.e., that there be a recursive function $f(x, y, z)$ such that for any numbers $i, j$ for which $\omega_{i}, \omega_{j}$ are complementary, $g(i, j, z)$ (as a function of the one variable $z$ ) maps $\omega_{i}$ into $\alpha, \omega_{j}$ into $\beta, \tilde{\omega}_{i}$ into $\tilde{\alpha}$ and $\tilde{\omega}_{j}$ into $\tilde{\beta}$." Several other results on universal sets and doubly universal pairs (as defined in [1]) are proved along the way.

\section{UNIVERSAL SETS}

1. Preliminary lemmas. We shall lean heavily on the following form of the iteration theorem:

THEOREM A. For any r.e. relation $M(z, x)$ there is a recursive function $t(x)$ such that for all numbers $i$ :

$$
x \in \omega_{t(i)} \leftrightarrow M(i, x)\left({ }^{5}\right) .
$$

We use Theorem A to obtain the following lemmas; in these lemmas (and throughout this paper) $\varnothing$ is the empty set and $N$ is the set of natural numbers.

LEMMA 1. For any r.e. set $A$ there is a recursive function $t(x)$ such that for every number $i$ :

(i) $i \in A \Rightarrow \omega_{t(i)}=N$,

(ii) $i \notin A \Rightarrow \omega_{t(i)}=\varnothing$.

Proof. Let $M$ be the set of all ordered pairs $(z, x)$ such that $z \in A$. Since $A$ is r.e., then $M$ is obviously a r.e. relation. Applying Theorem A we have (for every number $x$ )

$$
x \in \omega_{t(i)} \leftrightarrow M(i, x)
$$

Also:

$$
M(i, x) \leftrightarrow i \in A \quad[\text { by definition of } M]
$$

Hence

$$
x \in \omega_{t(i)} \leftrightarrow i \in A .
$$

(i) Suppose $i \in A$. Then for every $x, x \in \omega_{t(i)}$-i.e., $\omega_{t(i)}=N$.

(ii) Suppose $i \notin A$. Then for every $x, x \notin \omega_{t(i)}$-i.e., $\omega_{t(i)}=\varnothing$.

Our next lemma will be needed in Part II. We use the recursive pairing functions $J(x, y), K(x), L(x) . J(x, y)$ is a 1-1 recursive mapping of all ordered pairs of natural numbers onto the set of all natural numbers; $K(J(x, y))=x ; L(J(x, y))=y$.

(5) This is a special case of [1, Theorem 2, p. 68]. It is due to Kleene, being easily derivable from [2, Theorem XXIII, p. 342]. 
Lemma 2. For any two r.e. sets $A, B$ there is a recursive function $t(x)$ such that for every $i$ and $x$ the following conditions simultaneously hold:

(1) $i \in A \Rightarrow \omega_{K t i}=N$,

(2) $i \notin A \Rightarrow \omega_{K t i}=\varnothing$,

(3) $i \in B \Rightarrow \omega_{L t i}=N$,

(4) $i \notin B \Rightarrow \omega_{L t i}=\varnothing$.

Proof. By Lemma 1 , there is a recursive fiuncton $t_{1}(x)$ such that $i \in A \Rightarrow$ $\omega_{t_{1}(i)}=N$ and $i \notin A \Rightarrow \omega_{t_{1}(i)}=\varnothing$. By the same lemma, there is a recursive function $t_{2}(x)$ such that $i \in B \Rightarrow \omega_{t_{2}(i)}=N$ and $i \notin B \Rightarrow \omega_{t_{2}(i)}=\varnothing$. Define $t(x)$ to be $J\left(t_{1}(x), t_{2}(x)\right)$. Thən $K t i=t_{1}(i)$ and $L t i=t_{2}(i)$, and our result follows.

2. Generative sets. Consider a collection $\Sigma$ of r.e. sets. A number set $\alpha$ shall be called generative relative to $\Sigma$ if there is a recursive function $g(x)$ (under which $\alpha$ will be called generative relative to $\Sigma$ ) such that for every number $i$ for which $\omega_{i} \in \Sigma$, the following condition holds: $g(i) \in \alpha \leftrightarrow g(i) \in \omega_{i}\left({ }^{6}\right)$. If $\alpha$ is generative under $g(x)$ relative to $\Sigma$, then $g(x)$ shall also be called a generative function for $\alpha$ relative to $\Sigma$.

Relative to the collection of all r.e. sets, a generative function for $\alpha$ is a function which (in the terminology of Dekker) is completely productive for the complement of $\alpha$. And a productive function for $\alpha$ is a function which is generative for $\alpha$ relative to the collection of all r.e. sets which are disjoint from $\alpha$. [The verification of this is trivial; we need merely observe that for $\omega_{i}$ disjoint from $\alpha$, the condition " $g(i) \in \alpha \leftrightarrow g(i) \in \omega_{i}$ " simply says that $\left.g(i) \notin \alpha \cup \omega_{\imath}.\right]$ It is well known that a set is productive iff it is completely productive-i.e., iff it has a completely productive function. Thus a set $\alpha$ is generative relative to the collection of all r.e. sets iff it is generative relative to the collection of all r.e. sets which are disjoint from $\alpha$. Such a set $\alpha$ we shall simply call generative. Thus a generative set is one whose complement is productive (and hence differs from a creative set only in that it not necessarily recursively enumerable).

A set $A$ is called (many-one) reducible to $\alpha$ if there is a recursive function $g(x)$ which maps $A$ into $\alpha$ and $\tilde{A}$ into $\tilde{\alpha}$ (i.e., $A=g^{-1}(\alpha)$ ). And $\alpha$ is called universal if every r.e. set is reducible to $\alpha\left(^{7}\right)$.

It is shown in [4] that every universal set is generative. And it is also shown there that every generative r.e. set is universal. As we pointed out in [1], the preceeding statement holds even if $\alpha$ is not r.e. Hence $\alpha$ is generative iff $\alpha$ is universal.

We showed in [1] that a sufficient condition for $\alpha$ to be universal is that $\alpha$ be

(6) In the terminology of [1], this says that the complement of $\alpha$ is completely productive relative to $\Sigma$. The convenience of our present terminology will become more apparent as we proceed, especially in Part II where we extend this notion to ordered pairs.

(7) This is equivalent to every r.e. set being reducible to $a$ under a 1-1 function (cf. [4] or [1]), but the bi-uniqueness of the function shall play no rôle in the arguments of this paper. 
generative relative to the collection consisting of the empty set and all unit sets disjoint from $\alpha\left({ }^{8}\right)$. We now show

THEOREM 1. A sufficient condition for $\alpha$ to be universal is that $\alpha$ be generative relative to the collection consisting of just the 2 sets $N, \varnothing$.

To prove Theorem 1, we first note the following fact, whose proof is trivial.

Proposition 1. $\alpha$ is generative relative to the collection $\{N, \varnothing\}$ under $g(x)$ iff $g(x)$ is a recursive function which maps every index of $N$ onto a point inside $\alpha$ and every index of the empty set to a point outside $\alpha$.

Proof of Theorem 1. Let $g(x)$ be a (recursive) function under which $\alpha$ is generative relative to $(N, \emptyset)$. Then (by Proposition 1 ) for every $i$ :

(i) $\omega_{i}=N \Rightarrow g(i) \in \alpha$,

(ii) $\omega_{i}=\varnothing \Rightarrow g(i) \notin \alpha$.

Now let $A$ be any r.e. set which we wish to "reduce" to $\alpha$. Choose $t(x)$ as in Lemma 1. Then for every $i$ :

$$
\begin{array}{rlr}
i \in A & \Rightarrow \omega_{t(i)}=N & \\
& \Rightarrow \omega_{g t(i)} \in \alpha & {[\mathrm{by}(\mathrm{i})],} \\
i \notin A & \Rightarrow \omega_{t(i)}=\varnothing & \\
& \Rightarrow \omega_{g t(i)} \notin \alpha & {[\mathrm{by}(\mathrm{ii})] .}
\end{array}
$$

Then by (a), (b), the recursive functon $g t(x)$ is a (many-one) reduction of $A$ to $\alpha$.

3. Uniform reducibility. A collection $\Sigma$ of r.e. sets is uniformly reducible to $\alpha$ (as defined in [1, Chapter V]) if there is a recursive function $f(x, y)$ such that for every $i$ for which $\omega_{i} \in \Sigma$, the function $f(i, y)$ (as a function of the one variable $y$ ) is a reduction of $\omega_{i}$ to $\alpha$.

THEOREM 2. If the collection consisting of just the 2 sets $N, \varnothing$ is uniformly reducible to $\alpha$, then $\alpha$ is universal.

Proof. Let $N, \varnothing$ be uniformly reducible to $\alpha$ under $f(x, y)$. We show that $\alpha$ is then generative relative to $\{N, \varnothing\}$. Then it will follow from Theorem 1 that $\alpha$ is universal.

Let $g(x)=f(x, x)$. Clearly $g(x)$ is recursive (since $(f x, y)$ is). We show that $\alpha$ is generative under $g(x)$ relative to $\{N, \varnothing\}$. Result then follows by Theorem 1 .

(i) Suppose $\omega_{i}=N$. Then $f(i, y)$ reduces $\omega_{i}$ to $\alpha$. Let $y$ be any number. Then $y \in N \leftrightarrow f(i, y) \in \alpha$. Hence $i \in N \leftrightarrow f(i, i) \in \alpha$. But $i \in N$. Hence $f(i, i) \in \alpha-i$.e., $g(i) \in \alpha$.

(ii) Suppose $\omega_{i}=\varnothing$. Then $f(i, y)$ reduces $\varnothing$ to $\alpha$. Hence (for any $y$ ) $y \in \emptyset \leftrightarrow f(i, y) \in \alpha$. Hence $i \in \emptyset \leftrightarrow f(i, i) \in \alpha$. But $i \notin \emptyset$. Hence $g(i) \notin \alpha$.

REMARKS. (1) In [1] we proved (Lemma A, p. 105) that if $\Sigma$ is uniformly

(8) In fact, we showed that $\alpha$ is universal under a still weaker hypothesis; viz. that $\alpha$ be weakly productive. 
reducible to $\alpha$ under $f(x, y)$ and if for every set $A \in \Sigma$, the set $A^{*}$ (of all $i$ such that $f(i, i) \in A)$ is also in $\Sigma$, then $\alpha$ must be generative relative to $\Sigma$. Using this fact, we would immediately obtain Theorem 2 from Theorem 1 , since it is obvious that $N^{*}=N$ and $\emptyset^{*}=\varnothing$.

(2) Theorem 2 yields an alternative proof of a fact proved in [1, Theorem 9, p. 104], viz. that if the collection of all recursive sets is uniformly reducible to $\alpha$ then $\alpha$ is universal. For if this collection is uniformly reducible to $\alpha$, then obviously so is the smaller collection $\{N, \varnothing\}$.

This proof, unlike that of [1], does not make appeal to the fixed point theorem though, of course, it does use the iteration theorem.

\section{EFFECTIVE INSEPARABILITY}

4. Generativeness of ordered pairs. We now let $\Sigma$ be a collection of ordered pairs of recursively enumerable sets. A pair $(\alpha, \beta)$ of number sets shall be called generative relative to $\Sigma$ if there is a recursive function $g(x)$ (called a generative function for $(\alpha, \beta)$ relative to $\Sigma$-also a function under which $(\alpha, \beta)$ is generative relative to $\Sigma$ ) such that for every number $i$ for which $\left(\omega_{K i}, \omega_{L i}\right) \in \Sigma$, the following conditions both hold:

(1) $g(i) \in \alpha \leftrightarrow g(i) \in \omega_{K i}$,

(2) $g(i) \in \beta \leftrightarrow g(i) \in \omega_{L i}\left({ }^{9}\right)$.

Throughout the remainder of this paper we let $\Sigma_{2}$ be the collection consisting of the 2 pairs $(N, \varnothing),(\varnothing, N)$; we let $\Sigma_{3}$ consist of the 3 pairs $(N, \varnothing),(\varnothing, N)$, $(\varnothing, \varnothing)$; we let $\Sigma_{4}=\{(N, \varnothing),(\varnothing, N),(\varnothing, \varnothing),(N, N)\}$. We shall also consider the following conditions (which may or may not hold for a given $\alpha, \beta, g(x), i)$ :

$$
\begin{aligned}
& \mathrm{C}_{1}:\left(\omega_{K i}, \omega_{L i}\right)=(N, \varnothing) \Rightarrow g(i) \in \alpha, \\
& \mathrm{C}_{2}:\left(\omega_{K i}, \omega_{L i}\right)=(\varnothing, N) \Rightarrow g(i) \in \beta, \\
& \mathrm{C}_{3}:\left(\omega_{K i}, \omega_{L i}\right)=(\varnothing, \varnothing) \Rightarrow g(i) \notin \alpha \cup \beta, \\
& \mathrm{C}_{4}:\left(\omega_{K i}, \omega_{L i}\right)=(N, N) \Rightarrow g(i) \in \alpha \cap \beta .
\end{aligned}
$$

Now in place of Proposition 1 we need:

Proposition 2. Let $\alpha, \beta$ be a disjoint pair of number sets and $g(x)$ a recursive function. Then, under $g(x)$ :

(a) $(\alpha, \beta)$ is generative relative to $\Sigma_{2}$ iff for every i, conditions $\mathbf{C}_{1}$ and $\mathbf{C}_{2}$ both hold.

(b) $(\alpha, \beta)$ is generative relative to $\Sigma_{3}$ iff for every $i$, conditions $C_{1}, C_{2}, C_{3}$ all hold.

A pair $(A, B)$ is (many-one) reducible to $(\alpha, \beta)$ (as defined in [1]) if there is a recursive function $g(x)$ which simultaneously reduces $A$ to $\alpha$ and $B$ to $\beta$; equivalently, $g(x)$ maps $(A-B)$ into $(\alpha-\beta),(B-A)$ into $(\beta-\alpha), A \cap B$ into $\alpha \cap \beta$ and the complement of $A \cup B$ into the complement of $\alpha \cup \beta$. For a disjoint pair $(\alpha, \beta)$,

(9) In the terminology of [1], this says that the pair $(\tilde{\alpha}, \tilde{\beta})$ is completely doubly productive relative to $\Sigma$. 
a recursive function $g(x)$ is a reduction of $(\mathrm{A}, B)$ to $(\alpha, \beta)$ iff $g(x)$ maps $A$ into $\alpha$, $B$ into $\beta$ and the complement of $A \cup B$ into the complement of $\alpha \cup \beta$.

A pair $(\alpha, \beta)$ is called doubly universal (abbreviated D.U.) if every disjoint pair $(A, B)$ of r.e. sets is reducible to $(\alpha, \beta)$. [As shown in [1], this is equivalent to every disjoint pair of r.e. sets being reducible to $(\alpha, \beta)$ under a 1-1 function $g(x])$.

THEOREM 3. A sufficient condition for a disjoint pair $(\alpha, \beta)$ to be D.U. is that it be generative relative to $\Sigma_{3}$.

Proof. Let $g(x)$ be a recursive function under which $(\alpha, \beta)$ is generative relative to $\Sigma_{3}$. Let $(A, B)$ be any disjoint pair of r.e. sets which we wish to "reduce", to $(\alpha, \beta)$. Let $t(x)$ be as in Lemma 2. We show that the recursive function $g t(x)$ is a reduction of $(A, B)$ to $(\alpha, \beta)$.

(1) Suppose $i \in A$. Then $i \notin B$. Hence $\omega_{K t i}=N$ and $\omega_{L t i}=\varnothing$; i.e., $\left(\omega_{K t i}, \omega_{L t i}\right)=(N, \varnothing)$. Hence $g t(i) \in \alpha$ (by Proposition 2, part (b)).

(2) Suppose $i \in B$. Then $\left(\omega_{K t i}, \omega_{L t i}\right)=(\varnothing, N)$, so $g t(i) \in \beta$.

(3) Suppose $i \notin A \cup B$. Then $i \notin A$ and $i \notin B$, so $\left(\omega_{K t i}, \omega_{L t i}\right)=(\varnothing, \varnothing)$, hence $g t(i) \notin(\alpha \cup \beta)$.

By (1), (2), (3), $g t(x)$ is a reduction of $(A, B)$ to $(\alpha, \beta)$.

If a pair $(\alpha, \beta)$ is generative relative to a certain collection $\Sigma$, then it is obviously generative relative to any smaller collection $\Sigma^{\prime}$. Hence Theorem 3 yields an alternative proof of the fact (proved in [1] ) that a sufficient condition for a disjoint pair $(\alpha, \beta)$ to be D.U. is that $(\alpha, \beta)$ be generative relative to the collection of all disjoint pairs of recursive sets. [Again, this proof avoids appeal to the fixed point theorem.]

5. Effective inseparability. A pair $(A, B)$ can be recursively mapped into a pair $(\alpha, \beta)$ (as defined in [1]) if there is a recursive function $f(x)$ which maps $A$ into $\alpha$ and $B$ into $\beta$. This condition is, of course, considerably weaker than the existence of a reduction. [Even for a disjoint pair $(\alpha, \beta)$, a recursive mapping of $(A, B)$ to $(\alpha, \beta)$ need not map points outside $A \cup B$ to points outside $\alpha \cup \beta$.] As shown in [1, Proposition 4, Chapter IV, p. 98] if $(A, B)$ can be recursively mapped into a disjoint pair $(\alpha, \beta)$ and if $(A, B)$ is effectively inseparable, then $(\alpha, \beta)$ is in turn effectively inseparable. A fortiori, if $(A, B)$ is reducible to $(\alpha, \beta)$ and if $(A, B)$ is E.I. so is $(\alpha, \beta)$ (assuming $\alpha, \beta$ to be disjoint). So certainly every disjoint D.U. pair is effectively inseparable $\left({ }^{10}\right)$. It thus follows that if a disjoint pair $(\alpha, \beta)$ is generative relative to the collection of all disjoint pairs of recursive sets - or even relative to the collection $\Sigma_{3}$ - then $(\alpha, \beta)$ is effectively inseparable.

This fact comes fairly close to what we shall subsequently need for our metamathematical applications, but it is not strong enough; we need to prove the stronger fact that if a disjoint pair $(\alpha, \beta)$ is generative relative to the collection of

(10) We can take some E.I. pair of r.e. sets (whose existence is well known) and reduce it to the pair $(\alpha, \beta)$. 
all complementary pairs of recursive sets, then $(\alpha, \beta)$ is effectively inseparable. To this end we introduce a new notion.

We shall say that a pair $(\alpha, \beta)$ is semi-D.U. if every disjoint pair $(A, B)$ of r.e. sets can be recursively mapped into $(\alpha, \beta)$. Now, in order to show a disjoint pair $(\alpha, \beta)$ to be effectively inseparable, we do not have to show it to be D.U.; it suffices to show it to be semi-D.U. (by the same argument as footnote 10, reading "recursively map" for "reduce"). Now we can establish

THEOREM 4. If $(\alpha, \beta)$ is a disjoint pair which is generative relative to the collection of all complementary pairs of recursive sets-or even relative to the smaller collection $\Sigma_{2}$ - then $(\alpha, \beta)$ is effectively inseparable-in fact semiD.U.

Proof. In the proof of Theorem 3, steps (1) and (2) still hold under the weaker assumption that $(\alpha, \beta)$ is generative relative to $\Sigma_{2}$ rather than $\Sigma_{3}$. Step (3) will then, of course, fail, but steps (1) and (2) are all that are needed for the proof of semi-double universality rather than double universality.

We might note in passing, that if $(\alpha, \beta)$ obeys the hypothesis of Theorem 4 and if also $\alpha, \beta$ are both r.e. sets, then the pair $(\alpha, \beta)$ is not only semi-D.U., but actually D.U. This follows from Theorem 4 and the fact that every effectively inseparable pair of recursively enumerable sets is $\mathrm{D} . \mathrm{U}\left({ }^{\mathbf{1 1}}\right)$.

6. Uniform reducibility. A collection $\Sigma$ of ordered pairs of number sets is called uniformly reducible to a pair $(\alpha, \beta)$ (as defined in [1]) if there is a recursive function $f(x, y)$ (which is called a unifom reduction of $\Sigma$ to $(\alpha, \beta)$ such that for any number $i$ for which $\left(\omega_{K i}, \omega_{L i}\right) \in \Sigma, f(i, y)$ (as a function of the variable $y$ ) is a reduction of $\left(\omega_{K i}, \omega_{L i}\right)$ to $(\alpha, \beta)$. It is obvious that if $\Sigma$ is uniformly reducible to $(\alpha, \beta)$ then any sub-collection $\Sigma^{\prime}$ of $\Sigma$ is uniformly reducible to $(\alpha, \beta)$.

Let us consider a function $f(x, y)$. For any set $A$, let $A^{*}$ be the set of all numbers $i$ such that $f(i, i) \in A$. The following lemma was proved in [1,p. 118, Lemma B].

LEMMA. If $f(x, y)$ is a uniform reduction of $\Sigma$ to $(\alpha, \beta)$ and if for every pair $(A, B) \in \Sigma$ the pair $\left(A^{*}, B^{*}\right)$ is also in $\Sigma$, then $(\alpha, \beta)$ is generative relative to $\Sigma$.

From Theorem 4 and the above lemma, we now obtain our main result.

THEOREM 5. If $(\alpha, \beta)$ is disjoint and if the collection of all complementary pairs of recursive sets - or even if the smaller collection $\Sigma_{2}$-is uniformly reducible to $(\alpha, \beta)$ then $(\alpha, \beta)$ is E.I. - in fact semi-D.U.

Proof. Let $f(x, y)$ be a uniform reduction of $\Sigma_{2}$ to $(\alpha, \beta)$. Since $N^{*}=N$ and $\emptyset^{*}=\varnothing$, then $\Sigma_{2}$ has the closure property required in the hypothesis of the above lemma. Thus $(\alpha, \beta)$ is generative relative to $\Sigma_{2}$. Result then follows by Theorem 4 .

By a similar argument (using Theorem 3 in place of Theorem 4) we can assert

(11) This latter fact was first proved by Muchnik [9] and independently by Smullyan [1]. 
THEOREM 6. If $\Sigma_{3}$ is uniformly reducible to a disjoint pair $(\alpha, \beta)$ then $(\alpha, \beta)$ is D.U.

Again, we might note that if just $\Sigma_{2}$ is reducible to a disjoint pair $(\alpha, \beta)$ but if $\alpha, \beta$ are both r.e., then $(\alpha, \beta)$ is D.U.

7. On intersecting pairs. This section, though not needed for our metamathematical applications in $\S 8$, is presented to round out the purely recursive functiontheoretic aspects of the picture.

In Proposition 2 the requirement that $(\alpha, \beta)$ be disjoint is essential. For pairs whose intersection may be nonempty, we need to consider the following conditions $\mathrm{C}_{1}^{\prime}-\mathrm{C}_{4}^{\prime}$ in place of $\mathrm{C}_{1}-\mathrm{C}_{4}(\S 4)$ :

$\mathrm{C}_{1}^{\prime}:\left(\omega_{K i}, \omega_{L i}\right)=(N, \varnothing) \Rightarrow g(i) \in \alpha-\beta$,

$\mathrm{C}_{2}^{\prime}:\left(\omega_{K i}, \omega_{L i}\right)=(\varnothing, N) \Rightarrow g(i) \in \beta-\alpha$,

$\mathrm{C}_{3}^{\prime}$ : same as $\mathrm{C}_{3}$,

$\mathrm{C}_{4}^{\prime}$ : same as $\mathrm{C}_{4}$.

The following proposition (whose proof is again trivial) includes Proposition 2 as a special case.

Proposition 2'. Let $g(x)$ be a recursive function and $(\alpha, \beta)$ be any pair of number sets. Then for each $k=2,3,4,(\alpha, \beta)$ is generative (under $g(x, y)$ ) relative to $\Sigma_{k}$ iff for every $i$, conditions $\mathrm{C}_{2}-\mathrm{C}_{k}$ all hold.

Now, using Proposition 2 ' in place of Proposition 2, it is easily seen that Theorems 3 and 6 remain valid without the restriction that $\alpha, \beta$ be disjoint. Also, the following theorems can be proved by arguments similar to those already considered.

THEOREM 7.1. If $(\alpha, \beta)$ is generative relative to the collection of all ordered pairs of recursive sets - or even relative to $\Sigma_{4}$ - then every ordered pair of r.e. sets (whether disjoint or not) is many-one reducible to $(\alpha, \beta)$.

THEOREM 7.2. If the collection of all ordered pairs of recursive sets-or even if the smaller collection $\Sigma_{4}$ - is uniformly reducible to $(\alpha, \beta)$, then every ordered pair of r.e. sets is many-one reducible to $(\alpha, \beta)$.

\section{Metamathematical applications}

8. Effective inseparability of the nuclei of a theory. Consider now a consistent theory $(T)$ (not necessarily axiomatizable). Suppose that all recursive sets are uniformly definable in (T) (cf. introduction); let $\phi(x, y)$ be such that for any $i, j$ for which $\omega_{i}$ is recursive and $\omega_{j}$ is the complement of $\omega_{i}$, the formula $F_{\phi(i, j)}$ defines the set $\omega_{i}$ in $(T)$. Since $(T)$ is assumed consistent, this means that $F_{\phi(i, j)}$ represents $\omega_{i}$ and its negation represents $\omega_{j}$. If we now define $g(i, j)$ to be the Gödel number of the sentence $F_{\phi(K i, L i)}\left(\Delta_{j}\right)$, then it is obvious that $g(x, y)$ is a uniform reduction of the collection of all complementary pairs of recursive sets to the pair $\left(T_{0}, R_{0}\right)$. Hence, by Theorem 5 we have 
THEOREM 8. If $(T)$ is a consistent theory in which all recursive sets are uniformly definable, then the pair $\left(T_{0}, R_{0}\right)$ is effectively inseparable.

9. Definability of functions in $(T)$. If all recursive functions of one argument are definable in $(T)$ (in the sense of Tarski [3]) then certainly all recursive number sets are definable in $(T)\left({ }^{12}\right)$. Thus, if all recursive functions of one argument are definable in $(T)$ and if $(T)$ is consistent, then $\left(T_{0}, R_{0}\right)$ is recursively inseparable. We can easily obtain an "effective" analogue of this result as follows:

Let us say that all recursive functions of one argument are uniformly definable in $(T)$ if there is a recursive function $\phi(x)$ such that whenever a number $i$ is an index of a (general) recursive function $f(x)\left({ }^{13}\right)$, the number $\phi(i)$ is the Gödel number of a formula which defines $f(x)$ in $(T)$. Suppose now that $(T)$ is a consistent theory in which all recursive functions of one argument are uniformly definable. We assert that the hypothesis of Theorem 7 must then hold. For given an index of a recursive set $A$ as well as an index of its complement, we can certainly effectively find an index of the characteristic function of $A$ (i.e., there is a recursive function $h(x, y)$ such that whenever $\omega_{j}=\tilde{\omega}_{i}$, then $h(i, j)$ is an (r.e.)index of the characteristic function of $\left.\omega_{i}\right)\left({ }^{14}\right)$.

Then (by the hypothesis of Theorem 7) we can effectively find an index of a formula $F(x, y)$ which defines in $(T)$ this characteristic function. And then, of course, we can effectively find an index of the formula $F\left(x, \Delta_{1}\right)$, which defines $A$ in $(T)$. We thus also have:

THEOREM 9. If $(T)$ is a consistent theory in which all recursive functions of one argument are uniformly definable, then $\left(T_{0}, R_{0}\right)$ is effectively inseparable.

\section{REFERENCES}

1. R. M. Smullyan, Theory of formal systems, Annals of Mathematics Studies, No. 47, Princeton Univ. Press, Princeton, N.J., 1961.

2. S. C. Kleene, Introduction to mathematics, Van Nostrand, Princeton, N. J. 1952.

3. Alfred Tarski, Andrzej Mostowski and Raphael M. Robinson, Undecidabe theories, Studies in Logic and the Foundations of Mathematics, North-Holland, Amsterdam, 1953.

(12) For suppose $A$ is a recursive set; let $f(x)$ be its characteristic function, and let $F(x, y)$ define $f(x)$ in $(T)$. Then the formula $F\left(x, \Delta_{j}\right)$ defines $A$ in $(T)$.

(13) It makes no difference to this definition if by an "index" of a recursive function we mean an r.e. index (i.e., an index of the r.e. relation $f(x)=y$ ) or a "functional" index (i.e., a number $i$ such that $\left.f(x)=U(\mu y) T_{1}(i, x, y)\right)$. For it is proved in [1, Theorem 10, p. 92] that an r.e. index can be effectively found from a functional index, and conversely.

(14) For the relation $\left(x \in \omega_{z} \wedge y=1\right) \bigvee\left(x \in \omega_{w} \wedge y=0\right)$ is a r.e. relation of the four variables $x, y, z, w$. Then by the iteration theorem, there is a recursive function $h(z, w)$ such that for all numbers $i, j$ the number $h(i, j)$ is an index of the set of all pairs $(x, y)$ such that $\left(x \in \omega_{i} \wedge y=1\right) \bigvee$ $\left(x \in \omega_{j} \wedge y=0\right)$. But when $\omega_{j}=\left(\omega_{i}\right)^{\tilde{y}}$, this relation is precisely the set of all ordered pairs $(x, y)$ such that $f(x)=y$, where $f$ is the characteristic function of $\left(\omega_{i}\right)^{\sim}, \cdots$ i.e., when $\omega_{j}=\left(\omega_{i}\right)^{\sim}$, $h(i, j)$ is an index of the characteristic function of $\omega_{i}$. 
4. John Myhill, Creative sets, Z. Math. Logik Grundlagen Math. 1 (1955), 97-108.

5. R. M. Smullyan, Undecidability and recursive inseparability, Z. Math. Logik Grundlagen Math. 4 (1958), 143-147.

6. J. R. Schoenfield, Undecidable and creative theories, Fund. Math. 48 (1961), 171-179.

7. Putnam, H., Decidability and essential undecidability, J. Symbolic Logic. 22 (1957), 39-54.

8. Smullyan, R. M., Theories with effectively inseparable nuclei, Z. Math Logik Grundlagen Math. 6 (1960), 219-224.

9. A. A. Muchnik, Isomorphisms of systems recursively enumerable sets with effective properties, Trudy Moskov Mat. Obšč. 7 (1958), 407-412.

YESHIVA UNIVERSITY,

NEW YORK, NEW YORK 